\title{
Ensino de Testes de Software por meio de Digital Storytelling e Chatterbots
}

\author{
Luis Henrique C. $\operatorname{Rosa}^{1}$, Eduardo Lemos $^{1}$, Giliane Bernardi $^{1}, \operatorname{Roseclea~}_{\text {Medina }^{1}}$ \\ ${ }^{1}$ Universidade Federal de Santa Maria (UFSM) Caixa Postal 5082 - 97.105-900 - \\ Santa Maria - RS - Brazil \\ \{lhcr,giliane\}@inf.ufsm.br, \{elemos04, roseclea.medina\}@gmail.com
}

\begin{abstract}
The test activity is a critical element, considering that the costs associated with software failures are usually high. However, students in computing areas complete their training only with theoretical knowledge. The present study had the objective, through the technique of Digital Storytelling, to develop the play that was told the routine of a software tester; And through interaction with intelligent chatterbots to work the basics of area. A usability assessment was performed to analyze the effectiveness of the play and chatterbot. In the evaluation of the students the environment presents potential to assist the teaching of tests.
\end{abstract}

Resumo. A atividade de teste é um elemento crítico, considerando os altos custos associados às falhas de software. Contudo, a maioria dos estudantes de computação se formam apenas com o conhecimento teórico sobre a área. Buscando diversificar as estratégias para o ensino de testes de software, este artigo apresenta uma peça teatral desenvolvida em um Mundo Virtual 3D, baseada na técnica de Digital Storytelling, que "conta a história" da rotina de um testador de software. Ainda, por meio da interação com um agente conversacional (chatterbot) trabalharam-se os conceitos básicos de área. Com uma avaliação de usabilidade se analisou a efetividade desta abordagem, destacando-se o potencial do ambiente para auxiliar o ensino de testes.

\section{Introdução}

No desenvolvimento de software há uma preocupação relevante com a garantia de sua qualidade. A subárea da Engenharia de Software denominada Teste de Software é responsável pela verificação e validação do mesmo [Pressman 2011]. Estas etapas podem ser decisivas para o sucesso do projeto, pois falhas detectadas tardiamente são capazes de elevar consideravelmente os custos. Contudo, pesquisas mostram que a maioria dos bacharéis em computação somente adquirirão fluência nesta área quando necessitarem aplicá-la [Wangenheim 2009]. Isto porque o conteúdo é trabalhado, usualmente, apenas por meio de aulas expositivas, com escassez de atividades práticas e abordagens alternativas que motivem os estudantes [Pressman 2011; Silva et al. 2011].

Considerando essas colocações, Silva [2012] propôs um Mundo Virtual 3D que simula o ambiente de uma empresa de Teste de Software, desenvolvido na plataforma OpenSimulator [Opensimulator 2017], no qual foi implementado um Jogo Sério onde o estudante realiza atividades associadas às fases de teste para progredir no jogo. $\mathrm{Na}$ avaliação, dois aspectos importantes foram observados: primeiramente, que os 
estudantes apresentaram melhores interações e execução das atividades na presença do professor, que esclarecia dúvidas sobre o ambiente, regras do jogo e conteúdo, ocorrendo sensação de isolamento e "solidão" na sua ausência. O segundo aspecto observou que para a adequada utilização do jogo o estudante deveria dominar os conceitos iniciais do conteúdo, pois ele encontraria questões mais avançadas, tais como elaboração de planos e casos de teste.

Baseado nesta avaliação, o presente trabalho estendeu esse mundo, permitindo ao estudante iniciar sua interação com o ambiente discutindo conceitos iniciais e a importância da etapa de teste, ensejando a construção dos conceitos subsunçores que sirvam de base para agregar significado às informações avançadas sobre testes [Ausubel 2003]. Para a compreensão destes conceitos, foi utilizada a técnica de digital storytelling, que consiste em ensinar, capacitar e compartilhar conhecimento por meio de narrativas envoltas pela tecnologia [Yang et al. 2011]. Autores afirmam que a técnica mantém os estudantes mais concentrados, interessados e motivados, ajudando na melhor compreensão do conteúdo estudado [Santos e Daher 2008; Yang et al. 2011].

Para aplicação da técnica foi desenvolvido um teatro virtual, onde é apresentada uma peça adaptada do roteiro descrito em Rios e Moreira [2013], que discute a importância da realização de testes. Na peça foram utilizados agentes de software representando os funcionários (atores) de uma empresa de desenvolvimento de software composta por uma desenvolvedora e um testador, bem como um usuário, cliente da mesma. Ainda, buscando minimizar a "solidão" relatada pelos estudantes ao acessarem o ambiente na ausência do professor ou colegas, foi desenvolvido um agente conversacional (chatterbot) companheiro, que interage com os mesmos. As bases de conhecimento do agente foram desenvolvidas adotando o modelo de Benyon [2011], que sugere a criação de três modelos: Pessoa, Domínio e Interação.

Este artigo tem como objetivo central apresentar os resultados do desenvolvimento da peça, bem como do agente conversacional. Para isso o artigo está organizado com a seção 2 abordando o conceito de Digital Stoytelling, a utilização de Mundos Virtuais e Agentes de Software na educação são apresentados na seção 3, os Aspectos Metodológicos deste trabalho são trazidos na seção 4, na sequencia temos a caracterização da proposta na seção 5, já a Análise de Resultados é apresentada na seção 6, seguida das Conclusões na seção 7 e por fim as Referências bibliográficas.

\section{Digital Storytelling}

As histórias estão em todas as partes, para motivar os outros, transmitir informações, etc. Por isso, podem ser consideradas estratégias promissoras para atrair a atenção dos estudantes e vêm sendo usadas pelas escolas, que se beneficiam desse significativo recurso para educar nas mais diversas áreas do saber e graus de ensino do pré-escolar ao secundário [Junior et al. 2012]. Com o passar do tempo e a evolução das tecnologias, tornou-se possível contar essas histórias digitalmente, abrindo um novo campo para seu uso, facilitando sua criação e permitindo uma maturação em seus conteúdos.

Digital Storytelling, segundo Porter [2005], leva a arte antiga de narrativa ao uso de novas ferramentas para tecer contos pessoais usando, imagens, gráficos, música e sons misturados com a própria voz do autor. Autores afirmam que a técnica mantém os estudantes mais concentrados, interessados e motivados, facilitando a colaboração e a organização de ideias, bem como ajudando na melhor compreensão do conteúdo 
VI Congresso Brasileiro de Informática na Educação (CBIE 2017)

Anais do XXVIII Simpósio Brasileiro de Informática na Educação (SBIE 2017)

estudado [Van Gils 2005; Robin 2005, 2008; Santos e Daher 2008; Sadik 2008; Yang et al. 2011].

A técnica de Digital Storytelling pode ser organizada em quatro fases: 1) préprodução; 2) produção; 3) pós-produção; e 4) distribuição [Gere 2002; Ohler 2005; Robin 2005; Chung 2006; Kearney 2009]. A fase de pré-produção consiste em etapas que envolvem a definição das questões a serem abordadas com a história, a criação do roteiro, a implementação das narrativas orais e, por fim, do mapa da storytelling. Durante a fase de produção, a história é gravada, para que na fase de pós-produção essa gravação possa ser editada em uma versão digital, para então ser distribuída entre os participantes.

Usualmente, essas narrativas são criadas e executadas pelos participantes do processo, no caso aqui, estudantes. Contudo, este trabalho difere dessa metodologia de criação, pois a criação da storytelling foi responsabilidade do desenvolvedor do mundo virtual e os participantes da peça (atores) são agentes de software. Os estudantes, em um primeiro momento, são espectadores da peça, sendo que, após, podem interagir com o agente para esclarecer dúvidas sobre os conteúdos da disciplina ou do que foi discutido na encenação.

A próxima seção discute sobre Mundos Virtuais na Educação, destacando a inserção de agentes de software em tais ambientes.

\section{Mundos Virtuais e Agentes de Software na Educação}

Muitos trabalhos destacam as possibilidades dos mundos virtuais para a educação de nível superior [Wankel e Kingsley 2009; Savin-baden 2010; Vincenti e Braman 2011], aliando a tais ambientes abordagens de ensino como palestras, seminários, exibição de vídeos, simulações, performances virtuais, debates e interação com agentes de software.

Em Herpich et al. [2014], é apresentado um Mundo Virtual para apoiar o processo de ensino e aprendizagem de redes de computadores, utilizando agentes de software para elucidar conceitos sobre a área. Já em Salgado et al. [2013], Mundos Virtuais são utilizados para apoiar o ensino de lógica de programação. Neste trabalho, os autores utilizaram o ambiente OpenSimulator, integrado à ferramenta Scratch para trabalhar conceitos de lógica em um mundo tridimensional. Contudo, esses ambientes ainda apresentam problemas na manutenção da interação, sensação de isolamento e de solidão [Silva 2012]. Uma proposta para minimizar essas questões é a inserção de agentes inteligentes.

Sklar [2006] aponta a existência de três tipos de agentes inteligentes: os agentes pedagógicos, os peer learning agents (agente companheiro) e os agentes de demonstração. O agente companheiro busca simular o comportamento humano, atuando por meio da construção de um relacionamento com os estudantes [Boff 2008]. No caso deste trabalho, desenvolveu-se um agente inteligente do tipo companheiro conversacional (chatterbot), que consiste em um agente capaz de simular conversas inteligentes com um ou mais usuários humanos [Orlando e Giovanni 2008].

Dentre os exemplos de chatterbots de maior sucesso pode-se citar o A.L.I.C.E. [Alice 2017], sendo este a base do projeto PandoraBots, que consiste em um serviço de provedor de um software experimental de bots baseado no trabalho da comunidade de software livre [Pandorabots 2017]. Vemos a utilização desta plataforma na proposta do 
chatterbot ELAI, construído para apoiar a aprendizagem de conceitos sobre a área de redes de computadores em um mundo virtual 3D [Herpich et al. 2014]. Outro exemplo trata-se do trabalho de Bos [2014], que apresenta o chatterbot AGIMC, um guia inteligente de um Museu Virtual da História da Computação, também imerso em um Mundo Virtual 3D. Considerando a análise destes trabalhos correlatos ao que está sendo proposto, bem como do projeto PandoraBots, este serviço foi escolhido para ser utilizado no presente projeto. Na próxima seção apresentaremos os aspectos metodológicos que nortearam seu desenvolvimento.

\section{Aspectos Metodológicos}

A fase de desenvolvimento da peça teatral seguiu as diretrizes da estratégia de storytelling, iniciando com a definição da narração. Segundo Gancho [1991], existem cinco elementos principais que são componentes de uma narração: Enredo, definido como o conjunto de fatos de uma história; Personagens participantes do enredo, sendo eles fictícios ou não; o Tempo; O Espaço, podendo variar da época ou do tempo de duração dentro do local; e, por fim, o Narrador, sendo este o autor da história ou quem a conta.

Conforme apresentado na introdução deste artigo, a peça teatral desenvolvida é baseada no roteiro teatral descrito em Rios e Moreira [2013], denominado "Testar ou Não Testar - eis a questão", tendo como enredo a importância da realização da etapa de testes de software. Os personagens da peça, representados por agentes de software são o cliente, o desenvolvedor e o testador de software. O tempo pode ser considerado como sendo os dias atuais e a duração próxima ao período de uma aula de 50 minutos, utilizando o espaço de uma empresa de desenvolvimento. Por fim, o narrador não se faz presente como um personagem único, mas a junção dos três personagens presentes que "contam", cada um, sua parte da história.

Durante a fase de pré-produção da história, foi feito o estudo sobre o roteiro teatral de Rios e Moreira [2013], identificando seu tema e fazendo anotações com o intuito de adaptar à ideia desse trabalho. Na segunda fase, ou seja, a fase de produção, as alterações foram efetuadas e a história foi adaptada de maneira a se adequar ao tempo de aula. Já na fase de pós-produção foi feita a edição dos trechos da história, para que fossem inseridos como diálogos associados a cada avatar (agente) do mundo. Estes foram os responsáveis pela última fase, de distribuição, onde o ocorreu a "contação" da história pelos NPC, representando os atores da peça teatral. Inicialmente, o mundo contava somente com um terreno principal onde estavam as instalações do prédio da empresa de Teste de Software e onde era executado o Jogo Sério desenvolvido por Silva [2012]. Para este trabalho, optou-se pela construção de um novo terreno em anexo ao principal, com a construção de uma nova estrutura física, para a criação de um teatro e inserção dos agentes.

Para a avaliação, foram definidos como usuários-alvo 20 estudantes do curso de Mestrado em Tecnologias Educacionais em Redes de uma Universidade Federal. Optou-se por esses estudantes pelo fato dos mesmos terem o olhar de quem produz aplicações para educação. Outra justificativa para esta escolha deve-se ao fato dos estudantes selecionados cursarem a disciplina de Ambientes e Comunidades Virtuais de Ensino e Aprendizagem e estarem, no período da avaliação, iniciando atividades junto ao OpenSimulator. 
Para realização dos testes foi definido um roteiro com duas datas para visitação, uma fora do horário de aula e a outra durante um período da disciplina. As visitações foram planejadas para serem executadas de forma semelhante, permitindo uma avaliação posterior mais padronizada. Após assistirem à peça os estudantes apresentaram suas impressões, aos pesquisadores. Terminada essa interação, todos foram instruídos a interagirem com o agente. Por fim, foi solicitado que os estudantes respondessem a um questionário contendo 3 questões de perfil; 8 sobre a peça, NPC e sua estrutura e 6 sobre o chatterbot, por meio destas analisaram-se as percepções de cada um sobre o ambiente criado.

\section{Apresentação do Teatro e Agentes}

Esta seção destaca o desenvolvimento da infraestrutura do teatro, o roteiro, os agentes que atuam na peça (atores) e o agente conversacional companheiro. O teatro foi projetado com dois espaços, um interno e outro externo. No interior do teatro foi desenvolvido o espaço da plateia e o cenário (palco) para encenação da peça, exibidos na figura 1 .

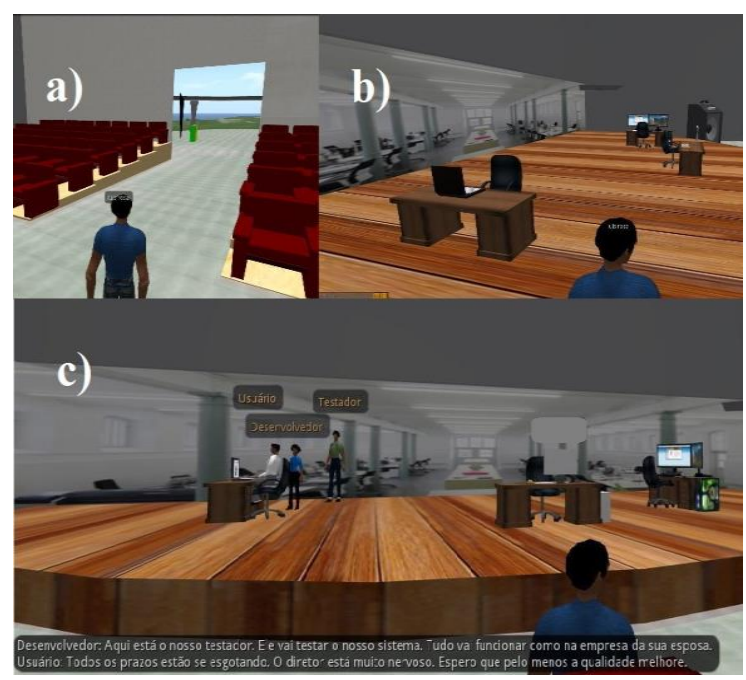

Figura 1. a) Espaço da plateia; b) cenário da peça; c) exibição da peça.

No hall de entrada, exterior do teatro, foi inserido o agente conversacional (chatterbot) companheiro, contendo uma base de conhecimento sobre conceitos de testes, com o objetivo de interagir com os estudantes na resolução de dúvidas sobre o tema, como mostra a figura 2 .

O agente se chama Zac e atua como um colega que ajuda o estudante a tirar dúvidas sobre o conteúdo por meio de interações pelo canal de chat do OpenSim. Para gerar a inteligência do agente foi utilizado o servidor de chatterbots PandoraBots, por meio da linguagem AIML. A base de conhecimentos foi estruturada de acordo com a arquitetura proposta por Benyon [2011], que sugere a criação de 3 (três) modelos: modelo de pessoa, domínio e interação.

Criou-se uma base de conhecimento (a) sobre o Mundo Virtual de Testes de Software; e (b) duas bases de interação, uma referente aos diálogos usuais de socialização e outra, a principal, contendo informações sobre os conteúdos específicos associados a conceitos de Testes de Software. O Modelo de Pessoa não foi desenvolvido neste primeiro momento. Para a construção do conhecimento sobre Teste de Software 
VI Congresso Brasileiro de Informática na Educação (CBIE 2017)

Anais do XXVIII Simpósio Brasileiro de Informática na Educação (SBIE 2017)

foram elencados alguns conceitos relevantes, considerando clássicos na literatura de Engenharia de Software [Pressman 2011; Sommerville 2012; Rios e Moreira 2013].

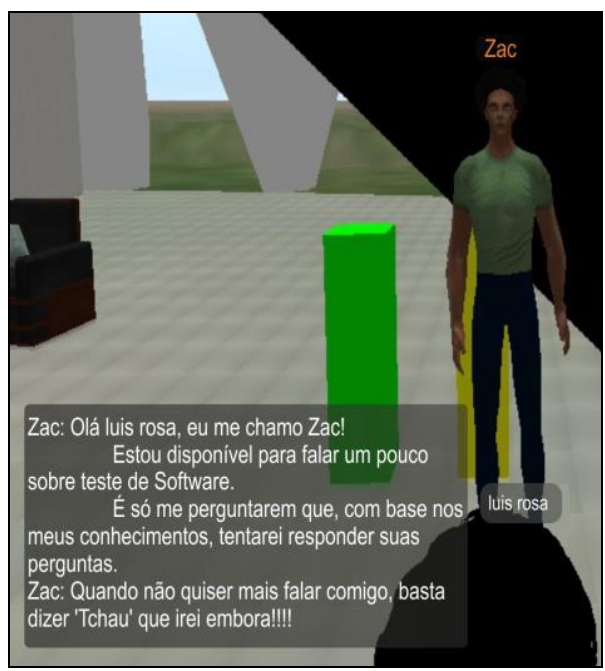

Figura 2. Interação como o agente.

Com todas as etapas concluídas, o ambiente desenvolvido foi apresentado para um grupo de estudantes, onde realizou-se uma avaliação de usabilidade, para analisar a efetividade dos NPC, da peça teatral e do chatterbot, sendo os resultados apresentados na próxima seção.

\section{Análise de Resultados}

Por meio do questionário foi montado o perfil dos avaliadores (20 respondentes) que são pessoas de ambos os sexos; distribuídos em diversas áreas de formação, sendo $50 \%$ da área de tecnologia, $25 \%$ da educação e $25 \%$ de outras áreas; dos quais $56 \%$ tinham experiência em ambientes virtuais 3D e de diversas faixas etárias.

Com relação às demais questões, buscavam investigar, sob o ponto de vista dos avaliadores, a efetividade do mundo criado, no que concerne à peça teatral e ao agente conversacional inserido. A figura 3 apresenta as questões relacionadas a avaliação da peça teatral e o gráfico resultante.

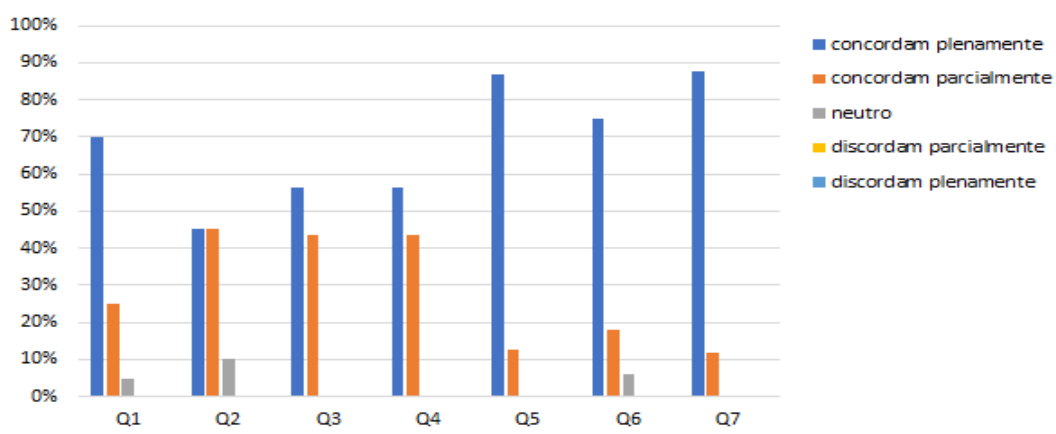

Q1: "Assistir uma peça teatral educacional foi uma experiência agradável?" Q2: "A peça teatral correspondeu às suas expectativas?"

Q3: "A mensagem educacional da peça teatral estava clara?"

Q4: "A peça teatral estava bem montada?"

Q5: "O espaço físico do teatro é agradável? "

Q6: "A peça pode ser considerada uma boa estratégia de aprendizagem?"

Q7: “A peça pode servir como ferramenta de auxílio ao ensino presencial?"

Figura 3. Gráfico das questões sobre a Peça. 
Nas questões de avaliação da peça, as respostas da questão Q1 apresentaram alto nível de satisfação com a proposta, corroborando com Osório et al. [2004] e Voss et al. [2013], que afirmam que as experiências em ambientes realísticos tendem a ser mais proveitosas. Quanto às expectativas com a peça e clareza da mensagem foi realizada a pergunta Q2. Com feedbacks positivos superiores a 80\% (concordo plenamente e concordo parcialmente) se comprova que o planejamento e montagem da peça foram bem-sucedidos.

As questões realizadas para avaliação da estrutura do ambiente e sobre a montagem da peça foram Q4 e Q5. A partir dos resultados foi possível constatar que assim como em Voss et al. [2013] e Dalsasso et al. [2014], a preocupação com a estrutura do ambiente faz com que o conteúdo trabalhado, neste caso a peça, agrade bastante aos usuários. Com resposta positiva a estrutura do ambiente e a peça é possível entender que a ambientação foi uma experiencia agradável, que proporcionou uma imersão tranquila no mundo.

Quando avaliado o seu potencial como ferramenta de apoio educacional e como apoio ao ensino presencial, a peça apresentou índices de respostas positivas superiores a $90 \%$. Os resultados da questão Q6 mostraram que a proposta de ambiente virtual 3D para o ensino se apresenta com vantajoso. Na questão Q7 foi avaliada da peça como ferramenta de auxílio ao ensino presencial, os resultados mostram que pode ser benéfica a sua utilização, conforme autores já ressaltaram a técnica de Digital Storytelling pode auxiliar na melhora da compreensão do conteúdo estudado [Santos e Daher 2008; Yang et al. 2011]. Com a aprovação da peça, é possível perceber que a proposta pode auxiliar no ensino, sendo uma alternativa para complementar o ensino expositivo em sala de aula.

O questionário ainda contou com uma questão aberta, para que os participantes tecessem comentários gerais sobre o ambiente avaliado, sendo que 12 dos 20 respondentes se manifestaram neste espaço. Foram apontadas melhorias na linguagem empregada e que a peça tenha seu tempo de duração reduzido, considerando que atualmente esse tempo é de 20 minutos. Contudo, todos mencionaram que a peça pode ser uma interessante alternativa para auxiliar no processo de construção do conhecimento.

Sobre a avaliação do agente (Figura 4), a questão Q1 obteve-se um bom nível de satisfação com os conhecimentos apresentados pelo agente. Os mais de $90 \%$ de aprovação mostram que foi realizado um bom mapeamento dos conceitos de Teste de Software. Na construção da base de conhecimento, quando perguntado Q2 e Q3 se verificou a aprovação dos participantes com o modo de interação do agente, $70 \%$ deles se mostram satisfeitos. Contudo os resultados demonstram que é necessária uma reformulação das tags de perguntas, para obtermos respostas mais precisas.

Para verificar o potencial educacional do agente foram realizadas as questões Q4 e Q5, os resultados positivos superiores a $80 \%$ mostram o agente como uma ferramenta com potencial para o melhoramento aprendizado. O que nos leva a perceber que com ajustes necessários este companheiro pode colabora para construção do conhecimento do estudante. Na Figura 4 são exibidos esses dados. 


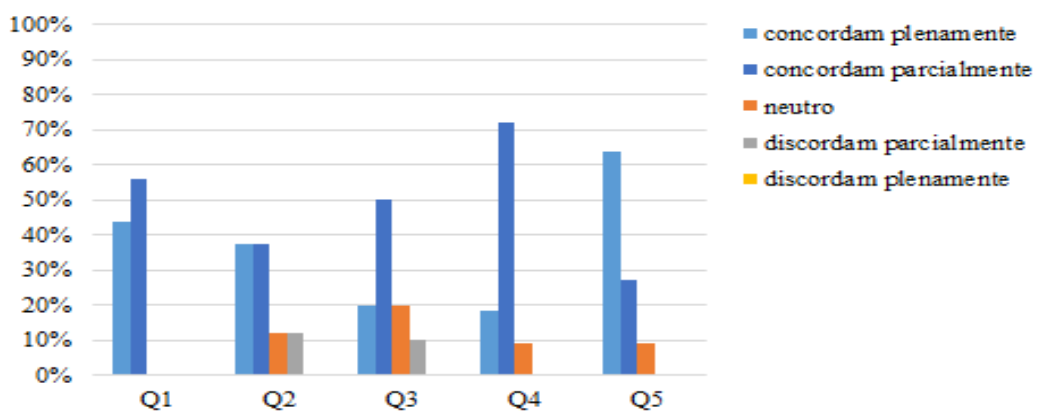

Q1: “O Agente Inteligente demonstrou bom conhecimento sobre Testes de Software?" Q2: "O Agente Inteligente respondeu às perguntas de forma satisfatória?" Q3: "As interações do Agente se mostraram satisfatórias? "

Q4: "O Agente Inteligente se mostrou uma boa estratégia de apoio a aprendizagem?" Q5: "O Agente pode servir como ferramenta de auxílio ao ensino presencial?"

Figura 4. Gráfico das questões sobre o agente.

Como já mencionado também havia um espaço para o estudante opinar sobre sua experiência com os agentes. Os relatos mostram que este tipo de interação pode ser facilmente utilizado como um apoio as aulas e como uma qualificada e interessante maneira de trabalhar os conteúdos. $\mathrm{Na}$ avaliação dos voluntários, o agente foi considerado uma boa estratégia de apoio ao ensino presencial, pois interagiu de forma satisfatória e que, com algumas alterações na maneira como as questões foram mapeadas haveria grande similaridade à interação entre humanos. Com essa avaliação também foi possível mapear outras formas de questionamento para as respostas da base de teste de softwares e, com isso, enriquecer mais a base de conhecimento dos agentes tornando-a mais robusta e assim atendendo mais adequadamente às interações.

\section{Conclusões}

A peça teatral apresentada neste artigo buscou, por meio da estratégia de Digital Storytelling, contar a história de um cenário de teste de software e, assim, trazer aos estudantes uma abordagem diferenciada para explorar estes conceitos. Com base nessas avaliações e nas observações realizadas, pode-se constatar que a peça apresenta potencial para ser utilizada com apoio ao ensino de teste de software, pois, segundo relatos, o conteúdo foi apresentado de forma compreensível e atrativo. Alguns comentários ainda apontaram que talvez mais contatos com a plateia tornariam a peça ainda mais interativa, causando maior envolvimento por parte do usuário.

Foi também inserido um agente conversacional companheiro (chatterbot) para interagir, elucidar dúvidas e mitigar os sentimentos de solidão e isolamento relatados pelos estudantes ao executarem as atividades sozinhos no ambiente. Por meio das avaliações percebeu-se as interações proporcionadas pela inserção do agente pode impulsionar a construção do conhecimento, possibilitando ao visitante possa aprender mesmo na ausência de professor ou um guia humano. Também se verificou que a simulação de comportamento humano pode ser uma boa solução para o problema de abandono ou solidão, contudo a avaliação mostrou que ainda são necessárias melhoras na forma como o agente entende os questionamentos para com isso proporcionar diálogos mais humanizados. 
VI Congresso Brasileiro de Informática na Educação (CBIE 2017)

Anais do XXVIII Simpósio Brasileiro de Informática na Educação (SBIE 2017)

\section{Referências}

Alice. (2017) A.L.I.C.E Artificial Intelligence Foundation. Acessível em http://alice.pandorabots.com/. Acessado em julho de 2017.

Ausubel, D. P., Novak, J. D., \& Hanesian, H. (1968) "Educational Psychology: A Cognitive View".

Benyon, D. (2011) Interface Humano-Computador. Brasil: Pearson, $2^{\mathrm{a}}$ ed.

Silva, T. G. (2012) Jogos Sérios em Mundos Virtuais: Abordagem para O Ensinoaprendizagem de Teste de Software. Dissertação (mestrado) em Ciência Da Computação - PPGI, UFSM. UFSM.

Boff, E. (2008) “Colaboração em Ambientes Inteligentes de Aprendizagem mediada por um Agente Social Probálistico”. Tese (Doutorado em Ciência da Computação) PPGC, UFRGS. Porto Alegre.

Bos, A. S. (2014) "A.G.I.M.C: Agente Inteligente Conversacional Como Guia Em Um Museu Virtual 3D da Computação". Dissertação (Mestrado em Ciência da Computação) - PPGI, UFSM. Santa Maria.

Chung, S. K. (2006) "Digital storytelling in integrated arts education". The International Journal of Arts Education, 4(1), 33-50

Gancho, C. V. (1991) “Como analisar narrativas”, São Paulo: Ática.

Gere, J. (2002) "Storytelling tools for the classroom". In J. Gere, B.-A. Kozlovich, \& D. A. I. Kelin (Eds.), By word of mouth: A storytelling guide for the classroom (pp. 18). Hawai.

Herpich, F., Nunes, F. B., Voss, G. B., Jardim, R. R., Medina, R. D. (2014) “Ambiente virtual imersivo para ensino em redes de computadores: uma proposta usandoAgentes Inteligentes", In: SBIE, Dourados, MS.

Junior, J. B; Lisbôa, E. S.; Coutinho, C. P. (2012) "Narrativas digitais na formação inicial de professores: Um estudo com alunos de licenciatura em pedagogia". Revista Teias, v. 13, n²7, pg 191-204. Pacific Resources for Education and Learning.

Kearney, M. (2009) "Towards a learning design for student-generated digital storytelling. In: The future of learning design conference". New South Wales, Australia: University of Wollongong.

Ohler, J. (2005) "The world of digital storytelling. Educational Leadership", 63(4), 4447.

Opensimulator. (2017) Disponível em: <www.opensimulator.org>. Acesso em 07/2017.

Orlando, P; Giovanni, F. (2008) "An integrated system, with natural language management, for the monitoring activities in e-learning environments". In: International conference on complex, intelligent and software intensive systems, Catalonia.

Osório, F. S.; Musse, S. R.; Santos, C. T. dos; Heinen, F; Braun, A; Silva, André T. da. (2004) "Ambientes Virtuais Interativos e Inteligentes: Fundamentos, Implementação e Aplicações Práticas”. XXIV CSBC - JAI 2004 (Jornadas de Atualização em Informática). Tutorial. Salvador, Bahia.

Pandorabots. (2017) Disponível em:http://www.pandorabots.com. Acesso em 06/ 2017. 
VI Congresso Brasileiro de Informática na Educação (CBIE 2017)

Anais do XXVIII Simpósio Brasileiro de Informática na Educação (SBIE 2017)

Porter, B. (2005) "Digitales: The art of telling digital stories". Denver: Colorado, USA: Bernajean Porter Consulting.

Pressman, R. S. (2011) Engenharia de Software: Uma Abordagem Profissional. Porto Alegre: Amgh, 7.ed.

Rios, E.; Moreira, T. (2013) Teste de Software. Rio De Janeiro: Alta Books. $3^{\text {a }}$ Ed.

Robin, B. R. (2005) “The educational uses of digital storytelling”. Retrieved November 1, 2011, from. http://digitalstorytelling.coe.uh.edu/getting_started.html.

Robin, B. R. (2008) "Digital storytelling: a powerful technology tool for the 21 st century classroom". Theory Into Practice, 47(3), 220-228.

Sadik, A. (2008) "Digital storytelling: a meaningful technology-integrated approach for engaged student learning". Educational Technology Research and Development, 56(4), 487-506.

Santos, V. G., e Daher, N. (2008) "Utilização de Storytelling como Ferramenta de Aquisição de Requisitos em Processo de Desenvolvimento de Software Apoiados em Modelos Ágeis: o uso apoiado no Extreme Programming”. E-tec, 1(1).

Savin-baden, M. A. (2010) "Practical guide to using Second Life in higher education". New York: McGraw-Hill, 2010.

Silva, T. S.; Müller, F. M.; Bernardi, G. (2011) "Panorama do Ensino de Engenharia de Software em Cursos De Graduação Focado em Teste de Software: uma Proposta de Aprendizagem Baseada em Jogos". Renote, V. 9, N. 2.

Sklar, E.; Richards, D. (2006) “The Use of Agents in Human Learning Systems". New York.

Sommerville, I. (2012) "Engenharia de software”. São Paulo: Pearson Addison Wesley, 9.ed. 2012.

Van Gils, F. (2005) "Potential applications of digital storytelling in education. In Paper presented at the 3rd twente student conference on IT". University of Twente, Department of Electrical Engineering, Mathematics and Computer Science.

Vincenti, G.; Braman, J. (2011) "Multi-user virtual environments for the classroom: practical approaches to teaching in virtual worlds". Hershey: Information Science Reference, 2011.

Voss, G. B.; Nunes, F. B.; Oliveira, T. B.; Medina, R. D. (2013) "TCN5 Desenvolvimento de um laboratório virtual de redes de computadores sensível ao contexto", In: WEI 2013.

Wangenheim, C. G.; Silva, D. A. (2009) "Qual conhecimento de engenharia de software é importante para um profissional de software? ”, In: FEES, 2.,2009, Fortaleza. Anais eletrônicos... Fortaleza: UFC.

Wankel, C.; Kingsley, J. H. (2009) "Education in Virtual Worlds”. Bingley: Emerald.

Yang, Y. T. C., \& Wu, W. C. I. (2012) "Digital Storytelling for Enhancing Student Academic Achievement, Critical Thinking, and Learning Motivation: a Year-long Experimental Study". Computers \& Education, 59(2), 339-352. 